\title{
La cucina dei vecchi Lussignani riprodotta nel suo dialetto (i veci piati de la cusina de Lussin)
}

\author{
Izvorni znanstveni rad \\ Original scientific paper
}

UDK 811.163.42'282.2(497.5)(210.7 Mali Lošinj):641

\begin{abstract}
Il dialettto lussignano fa parte del dialetto veneto come conseguenza del lungo dominio della Repubblica di Venezia sul territorio dell'Istria e del Quarnero. Questo dialetto è parlato solo, come dicono i lussignani, da coloro che da generazioni hanno sempre vissuto sull'isola e si sta estinguendo purtroppo insieme a loro. Le nuove generazioni parlano il lussignano solamente nella famiglia di origine. A volte capita che lo utilizzino per comunicare con i nonni, che rappresentano l'ultima generazione ancora legata al passato e che cerca di conservare, tramite la lingua, la propria identità culturale. La cucina dei vecchi lussignani assieme alle altre tradizioni, ha orgogliosamente mantenuto la propria individualità e trasmette inconsciamente lingua, usi e costumi di una volta.
\end{abstract}

Parole chiave: dialetto lussignano, cucina, Lussinpiccolo

Da quanto tempo non respirate il profumo forte e muschiato di un bosco, quell'odore intenso di mirto e di ginepro? Da quant' è che una ventata di aria di mare non vi fa chiudere gli occhi dal piacere, mentre l'essenza cristallina di alghe e di sale vi si propaga nel cuore? E quanti giorni sono passati dall'ultima volta che vi siete fermati a osservare l'arco che compie il sole nel cielo, i suoi riflessi nell'acqua, a percepire un silenzio che parla all'anima più di mille parole. L'isola verde di Lussinpiccolo aspetta proprio voi che amate la natura.

\section{INTRODUZIONE}

a cucina è un' arte sinestetica il cui messaggio passa attraverso sapori, profumi, sensazioni Ltattili, sensazioni visive e, in una certa misura, anche suoni. Storicamente è stata un importante strumento di identità di vari popoli e le differenze alimentari hanno costruito anche confini culturali tra vilaggi, città e nazioni.

La storia alimentare di un paese è anche la storia del paese stesso con il suo dialetto, tradizioni, modi di vivere e pensare della gente.

"Il nutrimento non è solo una collezione di prodotti soggetti a studi statici e dietetici, ma anche, nello stesso tempo, un sistema di comunicazioni, un corpo di immagini, un protocollo di usi, di situazioni, di comportamenti" (Barthes 1966: 29). 
Con la petuja, verže na ponfrịh, pašamête e altre pietanze, vogliamo introdurvi nella cucina lussignana di una volta e riscoprirne il dialetto, i cibi e le bevande tradizionali. E non c'è modo migliore di farlo che presentando le tipiche ricette del luogo.

Isola e sole, isola e mare. Chi viene a Lussinpiccolo compie un'immersione totale tra gente che il mare ce l'ha fuori dalla finestra, famiglie che vivono di mare e che il mare spesso e volentieri, se lo portano in tavola.

\section{LUSSINPICCOLO}

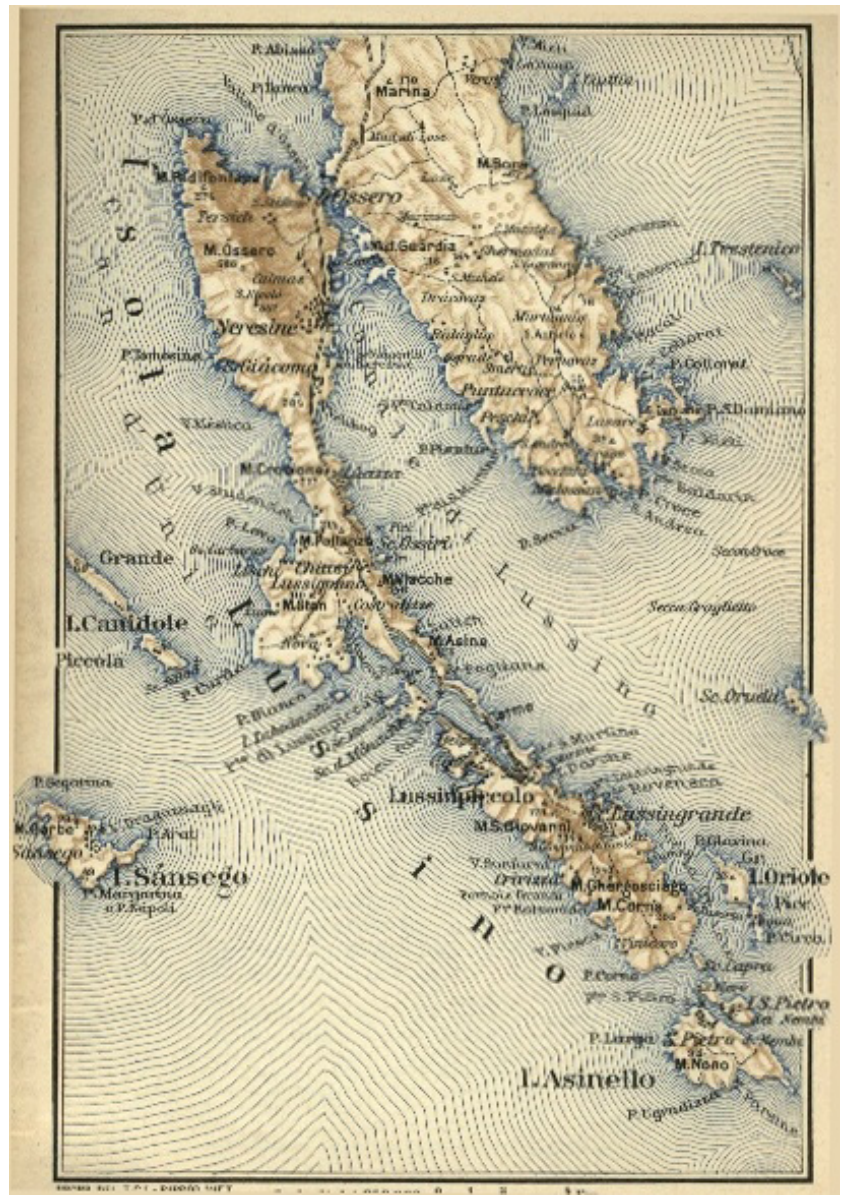

F. 1: L'isola di Lussinpiccolo

(http://xoomer.virgilio.it/histria/citta/lussino/lussinoindex.htm, 20.7.2010.)

Lussino è un'isola del Golfo del Quarnero, situato a nord del mar Adriatico. Giace al $44^{\circ}$, $27^{\prime}$ di latitudine N. e al $12^{\circ}, 15^{\prime}$ di longitudine E (Nicolich 1871: 13). Nell'antichità le due isole occidentali del Quarnero quelle cioè di Cherso e di Lussino, vennero separate dal ponticello chiamato Euripo, oggi detto Cavanella ${ }^{1}$.

L'isola è bagnata a occidente dal Quarnero (Kvarner, il mare esterno) e a oriente dal Quarnarolo (Kvarnerić, il mare interno).

In slavo la chiamavano "cavuada" dal veneziano "cavada" cioè scavata. 
Secondo il censimento del 2011 la città di Lussinpiccolo contava 5990 abitanti.

L'isola di Lussino era abitata già nell'età neolitica. Ciò ci viene confermato da numerosi reperti risalenti all'età della pietra ritrovati nella Grande Grotta (Vela Spilja²).

Furono gli Illiri, più precisamente i Liburni, che popolarono e costruirono case di pietra su tutte le isole del Quarnero.

Nel III e II sec. a.C. i Romani iniziarono a soppiantare gli Illiri. Dopo la caduta dell'Impero Romano d'Occidente, le isole di Lussino e Cherso passarono sotto la dominazione di Bisanzio.

$\mathrm{Nel} \mathrm{VI}$ sec.d.C. Ossero divenne sede episcopale con giurisdizione su tutto l'arcipelago. Alla fine del VI sec. ci fu una grande migrazione di popoli, ed in queste isole giunsero tribù di Croati, che appartenevano al grande gruppo degli Slavi del sud. Per tanti secoli Croati, Bizantini, Franchi, Veneziani e Ungheresi cercarono di imporre la propria egemonia su queste isole, finché nel 1409 i Veneziani ne divennero padroni assoluti per i prossimi quattro secoli.

"Insula Lussini" viene menzionata per la prima volta nei documenti del 1384. L'importanza di Lussinpiccolo aumentò sempre di più con la decadenza di Ossero, dal XV sec. in poi.

La presenza veneziana, che si protrasse per quasi quattro secoli, lasciò vistose tracce nell'architettura, nella lingua e nelle abitudini di vita della popolazione.

Dopo la caduta della Repubblica di Venezia (1797), questi territori furrono annessi per un breve periodo all'impero di Francia (1805) e successivamente alla Monarchia austriaca (1813).

Nel 1918, dopo la fine della I guerra mondiale e lo sfascio dell'impero austro-ungarico, questi territori entrarono a far parte del regno serbo-croato-sloveno, ma con il trattato di Rapallo (1920), le isole furono assegnate all'Italia, sotto il cui dominio rimasero fino al 1943" (Gasparotto 2004: 21).

Nel 1945 le isole entrarono a far parte della Jugoslavia e nel 1991 alla Croazia.

\section{CARATTERISTICHE DELLA PARLATA DI LUSSINPICCOLO}

Oggi la popolazione di Lussinpiccolo parla prettamente la lingua croata, anche se una parte, soprattutto gli anziani, usa per comunicare il lussignano.

Il lussignano è un dialetto che appartiene al tipo veneto giuliano (Deanović 1969: 255), ma che ha subito influenze di più lingue diverse: del tedesco austriaco ${ }^{3}$, dell'italiano e del croato, ma soprattutto del veneziano e del triestino, come si può riconoscere dall'uso di alcuni termini.

\subsection{FONETICA}

A differenza della lingua italiana il dialetto lussignano non distingue le vocali aperte e chiuse, il raddoppio delle consonanti e la " $z$ " sorda (zappa - zapa).

L'alfabeto è uguale a quello italiano eccetto i suoni che si riconoscono e distinguono nelle parlata lussignana che sono:

la /ts/: kolatsion / colazione - corrisponde all'africata dentale sorda /z/ come in zio, zeta,

il suono /ǵg/: ragǵo de šol / raggio di sole - corrisponde alla /g/ palatale dell'italiano gelo,

Si trova nella parte occidentale del monte Ossero.

Durante i 100 anni di governo austriaco la lingua ufficiale era l'italiano, e solo nelle zone rurali più isolate si parlava il dialetto croato. 
il suono /ń/: ńoki de patate / gnocchi di patate - corrisponde alla /n/ palatale italiana, scritta gn, bagno,

il suono /š/: škudella / tazza - corrisponde alla /š/ del croato škare (forbici),

il suono /Í/: saléra / saliera - corrisponde a gl(i) dell'italiano figlio,

il suono /ž/: kōža ti ga kužinạ? / cosa hai cucinato? - corrisponde alla /ž /del croato žlica (cucchiaio),

il suono /č/: čikara / tazzina da caffè - corrisponde alla /c/ italiana di cintura, celibe,

il suono /ć/: luontsić / pentolino - dovuto all'influenza della lingua croata, suono /k/: komoštre / catene del camino - corrisponde alla /k/ croata di kuća.

La pronuncia degli altri suoni segue le regole della lingua italiana.

\subsection{MORFOLOGIA (Abramić 1975: 7)}

\section{L'articolo}

L'uso dell'articolo davanti a determinati sostantivi qualche volta dipende da vere e proprie regole grammaticali e altre volte dall'uso della lingua parlata. Nel dialetto lussignano si distingue l'articolo determinativo e l'articolo indeterminativo.

L'articolo determinativo ha due forme al singolare:

- LA davanti ai nomi femminili: la Maria, la mann, / la mano - che è uguale come nella lingua italiana standard;

- $\quad$ EL davanti ai nomi maschili: el Karlo, el fio / il figlio - che è la particolarità nella parlata lussignana.

Al plurale, come nella lingua standard italiana fa LE per i nomi femminili ed I per i nomi maschili.

È interessante la forma plurale dell'articolo indeterminativo UNI e UNE come:

- Aggettivi indefiniti (alcuni, certe): $\underline{u} n i$ fjori, $\underline{u}$ ne škarpe

- Pronomi indefiniti e correlativi (gli uni, le une): uni tirava, altri molava

- Nella forma partitiva (dei, delle): go komprado uni libri; že dela une done

\section{I sostantivi}

I nomi sono di genere maschile o femminle, di numero singolare o plurale.

I nomi che al singolare terminano in -a sono di genere femminile ed al plurale prendono la desinenza -e; se sono di genere maschile, prendono al plurale la desinenza -i come: la šorela-le šorele; el poẹta-i poeti.

Ci sono nomi maschili e femminili che terminano al singolare per consonante e al plurale non seguono una regola come: la man - le man; el pịn - i pịni.

I nomi che al singolare terminano in - 0 sono generalmente di genere maschile ed al plurale prendono la desinenza -i come: el fio - i fjoi, el figo - i figi.

Alcuni nomi, sia maschili che femminili, terminano al singolare in -e e al plurale prendono la desinenza -i: el pje $\underline{e} d e$ - i pjedi, el pare - i pari. Terminano in -e ma si usano soltanto al singolare: la fáme, el pevere, el late; si usano soltanto al plurale: le molete, le tanaje (tanaglie), le mudande (mutande), le forfe (forbici).

\section{Aggettivo}

L'aggettivo accorda con il sostantivo nel genere e nel numero. Si divide in qualificativo e determinativo.

Alcuni aggettivi al singolare maschile terminano per consonante: bell, bon , sann.

L'aggettivo determinativo dimostrativo questo, $-a,-e,-i$, subisce un' elisione e diventa što $-a,-e,-i$, quando è seguito dal nome come ad esempio: što p $\underline{a} k o$, šta koža . 
I gradi dell'aggettivo seguono le regole della grammatica italiana: es. Óǵi že pjú káldo

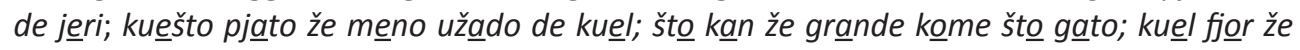
profumatišimo o la variante el pjū profumado.

Gli aggettivi possessivi sono:

singolare 1. persona: mín, mia, mii, mie

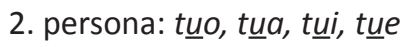

3. persona: šuo, šua, šui, šue

plurale 1. persona: noštro, noštra, noštri, noštre

2. persona: vošstro, vosštri, voštre, voštre

3. persona: - , - , de lori, de lore

L'aggettivo interrogativo ed esclamativo quale non si usa, si adopera soltanto che e quanto: es. Ke regallo ti me fara ? / Quale regalo mi farai?; Ke mižerja! - Che miseria!

Gli aggettivi numerali si usano come nella lingua.

\section{I pronomi}

I pronomi personali sono in funzione:

1. di soggetto: mi, ti , lui, ela, noi, voi, lori, lore

Prima persona ( singolare e plurale): mi kanto; noi kantemo

Seconda pesona( singolare e plurale): ti ti kanti; voi kante

Terza persona ( singolare e plurale): luii/ ela kanta; lori/e kanta.

Il pronome di seconda persona al singolare viene sempre raddoppiato, mentre il pronome personale di terza persona al plurale può avere anche questa forma: lori i kanta, lore le kanta.

\section{2. di complemento:}

- alla prima persona singolare mi corrispondono in funzione di complemento la forma mi e me, es. Ti ti me guardi; Ti vjên kon mi?

- alla seconda persona plurale ti corrispondono ti e te, es. Te go višsto; Parlavo de ti.

- alla terza persona singolare lui, lei corrispondono lo, la, el, la e luii, ela, ge, es. Lui el še pẹtina, ella la še pêtina; Mi lo go višto, Mi la go višsta; Kuešto že per lui e kuêšto že per êla; Mi ge digo šempre.

- alla prima persona plurale noi corrisponde ne, es. Noi ne guardẹmo in špećo; Ela ne penša šempre.

- alla seconda persona plurale voi corrisponde ve, es. Vọi ve lave ; Noi ve vedémo.

- alla terza persona plurale lori, lore corrispondono: li, le, lori, lore, ge, es. Lóri, i še lava šempre; mi li, le šeguo, Lui a bita kon lori, lore; Kuando li inkontro ge digo de andar študiár.

Le particelle ci evi per indicare un luogo non si usano.

Le particelle ne nelle espressioni di lui, di lei, di loro o nell'espressione neutra di ciò oppure aggiunta ad un gerundio, infinito, imperativo o assieme ad una particella pronominale non si usa. Esempi:

- Perke non ti me ga parlá? / Perché non me ne hai parlato?

- Gavendo pjatserr. / Avendone piacere.

- Fa košs' ti vol. / Fanne ciò che vuoi.

- Vado vía. / Me ne vado.

Le forme atone dei pronomi personali possono essere anche enclitiche. Es.

(Mi) Vole farme kuešto pjatser?

(Ti) Šon küa per parlarte.

(Si) Altsandoše el že žbrišă. 
(Vi) El koreveva čamandove.

(Lo, la) Guardilo, guardila.

(Gli) Non dirge.

(Li, le) Šentili, šentile.

I pronomi possessivi, dimostrativi, indefiniti, interrogativi ed esclamativi si usano come i corrispondenti aggettivi.

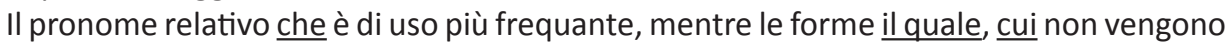
usate. Es.

- Go inkontra el tú amiko ke me ga šaluda.

- La kăža (nella quale) dọve štávo prịma... oppure: La kaža in ke ke štavvo prịma...

- $\quad$ El libbro de ke ke te diževo. Oppure: El libro ke te diževvo.

\section{Il verbo}

I verbi si raggruppano in tre coniugazioni. La prima coniugazione termina in -ar, la seconda in -er e la terza in -ir. I tempi composti si formano con l'aiuto dei verbi ausiliari êšer o gaver / essere e avere, ognuno dei quali ha una coniugazione propria.

\section{Verbi ausiliari ešer e gaver}

Con i verbi ausiliari nell'indicativo si usano soltanto i seguenti tempi: presente, passato prossimo, imperfetto, trapassato prosimo, futuro semplice e futuro anteriore. Nel dialetto mancano il passato remoto ed il trapassato remoto. Il congiuntivo si usa soltanto con gli ausiliari.

\section{Gaver}

Presente: mi go, ti ti ga, luii/el ga, ella/la/ga, noi gavemo, voi gave, lori/i/ga, lore/le/ga Imperfetto: mi gavevvo, ti ti gavevvi, lui/el/gaveva, ela/la/gavevva, noi gavevimo, voi gavevi, lori/i/gaveva, lore/le/gaveva

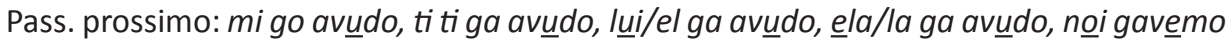
avudo, voi gave avuddo, lori/i/ga avudo, lore/le/ga avudo

Trapass. prossimo: mi gavevo avudo, ti ti gavevi avudo, luilel/gaveva avudo, ela/la/

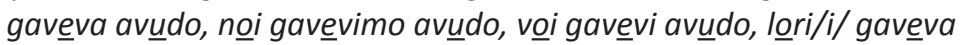
avudo, lore/le/gaveva avudo

Futuro: mi gavaro ti ti gavara luilel/ gavara, ela la gavara noi gavaremo, voi gavare, lori/i/gavara, lore/le/ gavara

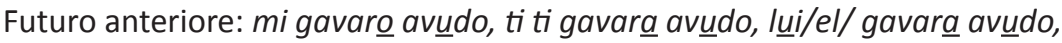
noi gavaremo avúudo, voi gavare avudo, lori/i/ gavara avudo

Del modo congiuntivo si usano soltanto il presente ed il passato:

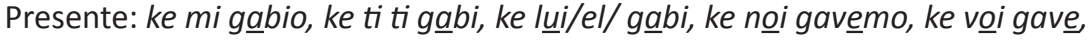
ke lori/i/gabi

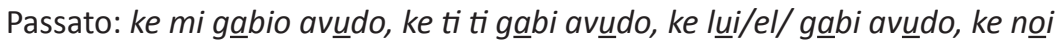

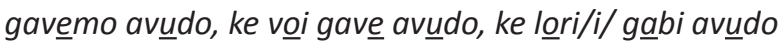

Del modo condizionale si usano tutti i due tempi, presente e passato: Presente: gavario, gavarii, gavaria, gavariimo, gavarii, gavaria

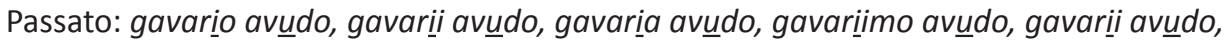
gavaria avudo 
Imperativo

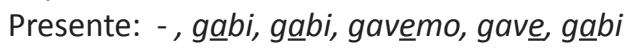

Futuro: -, gavara gavara gavaremo, gavare gavara

Infinito

Presente gaver

Passato gaver avudo

Participio

Presente -

Passato avudo

Gerundio

Presente gavendo

Passato gavendo avudo

Nell'uso corrente del dialetto si adoperano quasi esclusivamente i tempi del modo indicativo, participio passato e condizionale; gli altri modi sono poco frequenti.

\section{Ešer}

Presente: mi šon, ti ti že, luii/el/že, noi šemo, voi še, lori /i/že

Imperfetto: mi jêro, ti ti jerri, luii/el/ jẹra, noi jẹrimo, voi jeri, lori/i/ jera,

Passato prossimo: mi šon šta/do/, ti ti že šta/do/, luii/el/že šta/do, noi

šemo štadi, voi še štadi, lori/i/ že štadi

Trapassato prossimo: mi jero šta/do/, ti ti jerri šta/do/, luilel/ jerra šta/do/, noi jerimo štadi, voi jêri štadi, lori/i/ jera štadi

Futuro: mi šaro, ti ti šara, luilel/ šara, noi šaremo, voi šare, lori/i/šara

Futuro anteriore: mi šaro šta/do/, ti ti šara šta/do/, luilel/ šara šta/do/, noi šaremo štadi, voi šare štadi, lori/i/ šara štadi

Del modo congiuntivo si usano solo il presente ed il passato:

Presente: ke mi šio, ke ti ti šii, ke luil/el/ šia, ke noi šemo, ke voi še, ke lori/i/ šía

Passato: ke mi šio šta/do/, ke ti ti šii šta/do/, ke luii/el/ šía štáado, ke noi šemo štadi, ke voi še štadi, ke lori/i/ šia štadi

Condizionale

Presente: šarịo, šariii, šarịa, šariimo, šariii, šaria

Passato: šarịo šta/do/, šarịo šta/do/, šarịa šta/do/, šariimo štadi, šarịi štadi, šarịa štadi

Imperativo

Presente: - , šii, šia, šemo, še ši

Futuro: - , šara, šara, šaremo, šare, šara

Infinito

Presente e ěser

Passato êšer štado

Participio

Presente -

Passato štado

Il gerundio non si usa. 


\section{Verbi regolari della prima coniugazione -ar}

\section{Parlar}

Indicativo

Presente: parlo, parli, parla, parle mo, parle, parla

Passato prossimo: mi go parla/ parlado, ti ti ga parla/ parlado, luilel/ ga parla/ parlado, noi gavemo parla/ parlado, voi gave parla/ parlado, lori/i/ ga parla/ parlado

Imperfetto: parlavvo, parlávi, parláva, parlavimo, parlávi, parlavva

Trapassato prossimo: gavevo parla, gavevi parla gavevva parla gavevimo parla gavevi, parla gaveva parla

Il passato remoto ed il suo tempo composto non si usano.

Futuro: parlaro, parlara parlara parlaremo, parlare parlara

Futuro anteriore: gavaro parla , gavara parla , gavara parla gavaremo parla gavare parla gavara parla

Il modo congiuntivo del dialetto lussignano, a differenza degli altri, non è usato; invece si adoperano sempre i tempi dell'indicativo o condizionale.

\section{Condizionale}

Presente: parlario, parlarii, parlaria, parlariimo, parlarii, parlaria

Passato: gavario parlá, gavarii parla gavaria parla gavariimo parla gavarii parla gavarịa parla

Imperativo

Presente: - , parla, parli, parlemo, parla, páali

Futuro: - , palara, parlara, parlaremo, parlara, parlara

Infinito

Presente parlar

Passato gaver parla / parládo

Participio

Presente non si usa

Passato parla / parládo

Gerundio

Presente parlando

Passato: gavendo parla /parlädo

\section{Verbi regolari della seconda coniugazione -er}

\section{Škriver}

Indicativo

Presente: škrịvo, škrịvi, škrịve, škrivemo, škrive škrive Imperfetto: škrivevo, škrivevi, škrivevva, škrivevino, škrivevi, škriveva

Passato prossimo: go škrito, ga škrito, ga škrito, gavemo škrito, gave škrito, ga škrịto Trapassato prossimo: gavevo škrito, gavevi škrịto, gaveva škrito, gavevimo škrịto, gavevi škrito, gaveva škrito.

Futuro: škrivero, škrivera, škrivera, škriveremo, škrivere, škrivera 
Futuro anteriore: gavaro škrịto, gavara škrịto, gavara škrịto, gavaremo škrịto, gavare škrito, gavara škrịto

Condizionale

Presente: škriverịo, škriverii, škriverịa, škriveriiimo, škriverii, škriveria

Passato: gavarịo škrịto, gavarịi škrịto, gavarịa škrịto, gavarịimo škrito, gavarii škrịto, gavaria škrịto

Imperativo

Presente: - , škrivi, škrive, škrivemo, škrive, škrive

Futuro: - , škrivera škrivera škriveremo, škrivere, škrivera

Infinito

Presente škriver

Passato gaver škrito

Participio

Presente -

Passato škrito

Gerundio

Presente: škrivendo

Passato: gavendo škrito

\section{Verbi regolari della terza coniugazione -ir}

Šentir

Indicativo

Presente: šento, šenti, šente, šentimo, šenti, šente

Imperfetto: šentivo, šentivi, šentiva, šentivimo, šentivi, šentiva

Passato prossimo: go intežo, ga intežzo, ga intežzo, gavemo intežo, gave intežo, ga intežo Trapassato prossimo: gavevvo intežo, gavêvi intežo, gavevva intežo, gavevimo intežo, gavevi intežo, gavevva intę̌̌o

Futuro: šentiro, šentira, šentira, šentiremo, šentire, šentira

Futuro anteriore: gavaro intežzo, gavara intęžo, gavara intežo, gavaremo intežzo, gavare intežo, gavara intežo

Il congiuntivo non si usa.

Condizionale

Presente: šentirio, šentirii, šentirịa, šentiriimo, šentirii, šentirịa

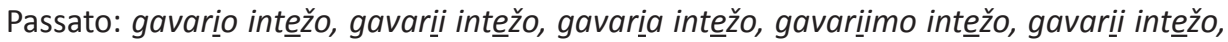
gavarịa intežo

Imperativo

Presente: / , šenti, šente, šentimo, šenti, šente

Futuro: - , šentira šentira, šentiremo, šentire šentira

Infinito

Presente šentir

Passato gaver intežo 
Participio

Presente -

Passato intežo

Gerundio

Presente: šentido

Passato: gavendo intęžo

\section{Verbi irregolari}

I verbi irregolari seguono lo schema dei verbi irregolari della lingua.

Es.

\section{Andar}

Presente: vado, $v a, v a$, andemo, ande, $v a$

Dir

Presente: diggo, dǐži, diže, dižẹmo, dịže, dịže

\section{Voler}

Presente: vojo, vol, voll, volemo, vole, vol

\section{Uso dei modi e dei tempi}

\section{Modo indicativo}

Il presente indica un'azione, uno stato o un modo di essere che si verificano o sussistono nel momento in cui si parla: kuel putel nuda. Si usa anche per indicare stato o azione del futuro imminente: štašera vado in čine.

Il futuro semplice indica un fatto che, nel momento in cui si parla o scrive, deve ancora avvenire o giungere a compimento: ti partira fra djẹtsi ǵorni; ašpeteremo per ńénte. II futuro semplice può anche riferirsi ad avvenimento presente o passato, quando chi parla vuole esprimere dubbio o incertezza: non ti gavara miga una pištola adọšo?

II futuro anteriore indica un evento futuro che sarà già compiuto o dovrà essere compiuto prima che se ne realizzi un altro, anch'esso futuro: kuando ti gavara finido de lavorar te veńiro prender.

L'imperfetto indica un'azione passata considerata nel suo svolgimento e esprime la "durata" di un azione nel passato: andạvimo e veńivimo šentsa fermarše maj.

Il passato prossimo indica un fatto avvenuto in un passato molto recente oppure un fatto avvenuto in un passato anche molto lontano, ma i cui effetti perdurano ancora nel presente:

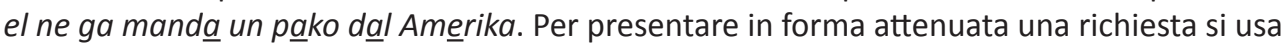
il passato prossimo al posto del presente: šon veńudo domandarte un pjatser

II trapassato prossimo esprime un fatto avvenuto prima di un altro fatto del passato e ad esso collegato: kuel ǵorno me jerra andádo túto per trešo.

\section{Modo condizionale}

Nelle proposizioni indipendenti il presente serve ad esprimere dubbio, incertezza o possibilità; con lo stesso significato si usa il condizionale passato: mi dirịo ke le kože non že andáa kožị kôme ke i dịže; ki gavarịa mái dẹto!?

\section{Modo imperativo}

Esprime comando ed esortazione; una forma particolare ha l'imperativo negativo con l'aggiunta del verbo štar: non šta korer; non šta mańar šta roba. 


\section{Modo infinito}

L'infinito presente esprime l'idea generica del verbo. L'infinito passato indica un'azione compiuta al passato: šo de gaver študjạ; šo de gaver fato mall.

\section{Participio}

Il participio passato quando è unito all'ausiliare ešer si accorda col soggetto nel genere e nel numero: $i$ že partidi; quando è unito all'ausiliare gaver rimane invariabile rispetto al soggetto gavemo mańado.

\section{Gerundio}

Il gerundio si usa nelle proposizioni dipendenti di vario genere: tornando a káža lo go incontra.

\section{L’apostrofo}

É interessante un modo efficace della vecchia maestra lussignana, Maria Straulino, per far ricordare ai bambini cos'è l'apostrofo:

"Un giorno, l'articolo camminava in mezzo al deserto; a un certo punto la vocale si perse e non potè più essere ritrovata. La "elle" allora si disperò a tal punto per aver perso la compagna, che pianse. L'apostrofo è la lacrimuccia che spuntò alla "elle"”" (Lussino 29: 62).

\section{CIBI E BEVANDE TRADIZIONALI}

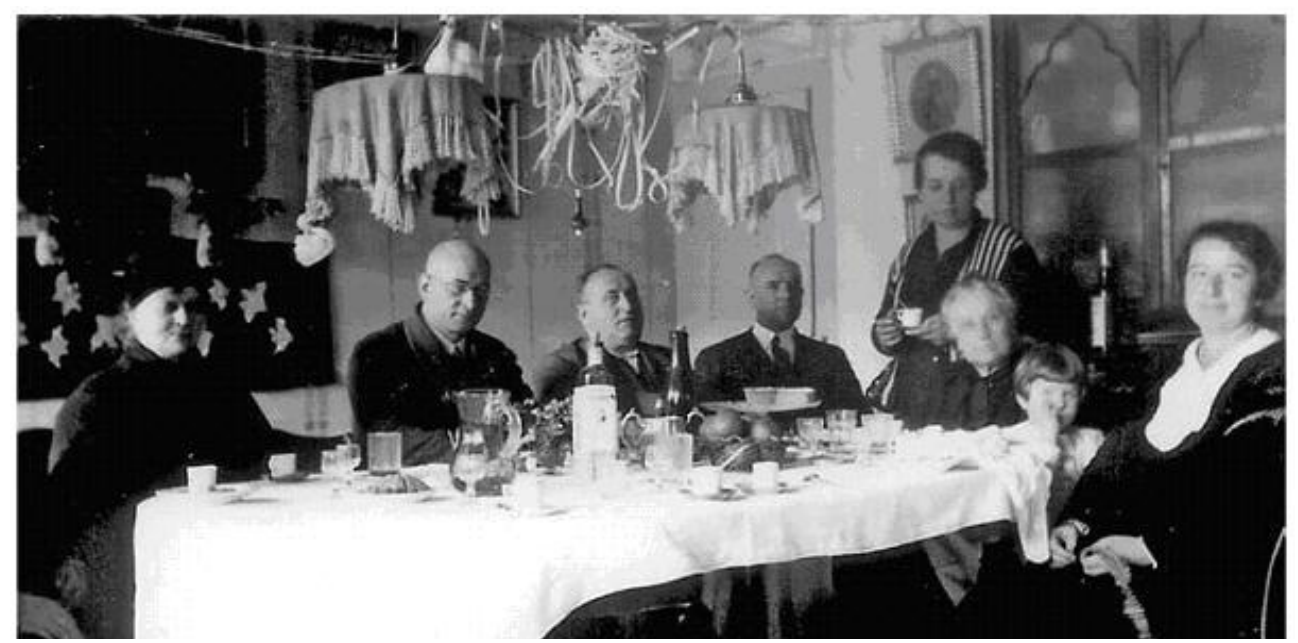

F. 2: Lussignani a tavola (Lussino 32: 31)

Cosa accade in luoghi, come Lussino, dove il mare incontra la terra, dove ci sono grandi spiagge e dolci colline, dove la cultura dei contadini si intreccia con quella dei pescatori? Dove lo scambio proficuo giunge fino in tavola, con ricette che spaziano in due mondi, all'apparenza tanto diversi, ma che sull'isola hanno un legame antico, ben saldo. I due volti dell'isola: la campagna e il mare si incontrano in cucina dove si incontrano sfumature raffinate nella mescolanza di ingredienti apparentemente contrastanti. L'inventiva delle donne lussignane addette ai fornelli risale a tanto, tanto tempo fa. 
“La mensa, come è ben naturale, era frugante e semplice, qualche po' di pesce, qualche verdura, od i legumi formavano il principale nutrimento, ma vi era condito da tranquilità d'animo, da reciproco affetto, e della benedizione d'una bamboleggiante prole" (Nicolich 1871: 201).

\subsection{CIBI TRADIZIONALI}

\subsubsection{PRIMI PIATTI}

\section{Brodo brustolà}

Cibo leggero, dietetico.

Ingredjenti: due kućari de farina, due kućari de buro, due ovi, un littro de akua

Preparatsjon: In una téća še friževa la farina nel buro; kuando ga ćapa un poko de kolor še mête un lítro de akua e še ašpetta ke boje. De parte še žbate i ovi intjeri kon un pokko de šal e poj še verša tuto in brodo, šempre žbatendo e fatsendo atencjon ke non vjẹn le grudvice (grumi).

Še prepara in pjati le pašamete (fette di pane abbrustolito), šal, pevere (pepe) e še veršava el brodo bojente.

\section{Budin de patate}

II budino si usava fare spesso per prantso perché era un piatto semplice da preparare.

Ingredjenti: patate, buro, ovi, mežo bićer de late, prošuto

Preparatsjon: Še boje le patate, še ašpeta ke le še rafreda e poj še buta kuatro ǵáli de ovo, el buro škuajájo (amorbidito), še mete late, el šal, pevere e prošuto tajáado in fin e ankora meter el bjanko del ovo.

Še onže (ungere) un štampo kon el buro e pan gratạ e še mete dentro el impašto. Bižonja lašar in forno per kuarantatsinkue minuti a bańomarịa. Kuando že pronto še mête šul budin el buro e prošuto tajado a petsetini.

\section{Kaputsi garbi}

Piatto invernale che piace molto ai lussignani.

Ingredjenti: un killo de kaputsi garbi, ojo, tsivola, farinna, luganige

Preparatsjon: še deve lavar ben i kaputsi, še prepara un šofrito de ojo kon dú kućarini de farina e tsivola tajada. In pińata še mete alora anke i kaputsi aǵunǵendo anke šal kuanto

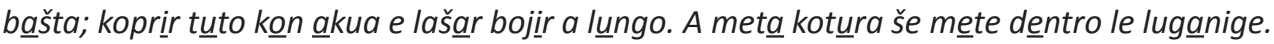

\section{Krapfen}

Non è un dolce. Si usava fare come primo piatto.

Ingredjenti: un killo de patate, kuatrotsento grami de farina, kuatro ovi, venti grami de levito per ńoki, buro, fegatini, tsukero

Preparatsjon: Še boje e páša le patate, še mete un poko de farina, i ǵali de ovo, el ĺvevito ke prịma el še škuaja in una čikareta kon un poko de late tjẹpido. Kon kuešto impašto še fa

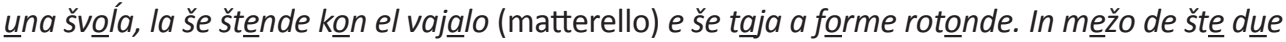
forme še mête un kućár de fegatini de polo šofriti nel buro. I še kjüde ben e i še friže in ojo.

\section{Noketi de pan per brodo}

Molto semplice da fare e sostanzioso. Siccome a Lussino non si buttava via niente allora si poteva fare anche con el pạn véćo (pane raffermo).

Si usava dire anche un ninin (giusto un po'). 
Ingredjenti: kuatro ovi, kuatro kućari de late, pạn grata, peršemolo (prezzemolo)

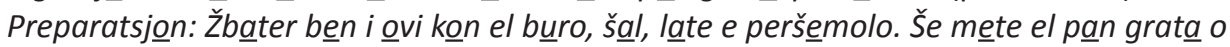

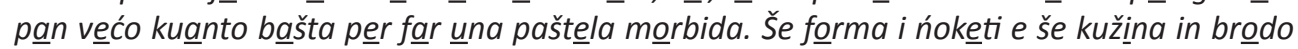
per kuinditsi minuti.

\section{Ńoki lušińani}

I ńoki si mangiavano di domenica per pranzo. Si condivano con vari sughi di carne o fegato.

Ingredjenti: due ovi, due kućari de buro, farina, tre o kuatro kućari de late

Preparatsjon: In una terina še žbate i ovi, el buro, šal, farina e late. Kon što impasšto še fa

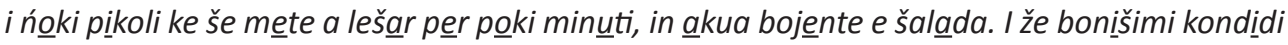
kon šugo de karne.

\section{Mineštra de verdura}

Usando gli ingredienti poveri, si sono perfezionati nel tempo molti tipi di minestre, zuppe, pietanze tipicamente isolane, che costituivano il pasto serale. Si trattava di mineštra de verdure in molte varianti, con lenticchie, fagioli, piselli, fave, ceci e orzo, alimenti che si adattavano ad essere conservati secchi e che, cotti separatamente, costituivano ciascuno la base per una diversa zuppa, in modo da variare il sapore. Tutte partivano da un soffritto di aglio e cipolla nell'olio d'oliva e finivano, servite calde, con pesce fritto.

Ingredjenti: blitva, favva, biži, patate, fažoj, karota, tsivola, peršemolo, šeleno

(sedano), ojo de oliva, šal, pevere

Preparatsjon: Frižer šul ojo i fažoj, la fạva, i biži, le patate tajáade a dadini e altri ingredjenti tajadi in fin. Aǵunńer a kua, šal e pevere e kužinar a fogo lento per un ora.

\section{Oržo e fažoj}

"Oržo e fažoj - mineštra de poveri fjoil"

Un giorno si mangiava oržo e fažoj, l'altro riži e bǐži e poi per cambiare pášta e fažoj, e così da capo.

Ingredjẹnti: oržo, fažoj, tsivolla, dụe kućari de farinna, luganige, un littro e mežo de akua

Preparatsjon: še kužina in akua šentsa šal el oržo e i fažoj ( i fažoj e el oržo še devve mêter a bańomarịa la šera prịma). Še fa un šofrito de tsivola, peršemolo e farina, še verša in akua de kotura dêla mineštra, e še fa bojịr inšjẹme kon le luganige. Ala fine še mête un poko de

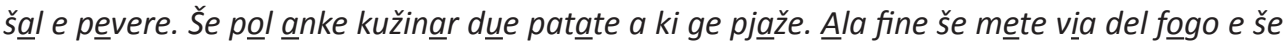
aǵunne un kućar de ojo de oliviva e atseto.

\section{Riži e biži}

Ingredjẹnti: bǐži, mežza tsivola, rǐži, peršemolo, büro

Preparatsjon: Še friže la tsivola in ojo de olivva, še aǵunǵe i biži freški, peršemolo, šal e un poko de ákua. Kuando i bǐži šara tẹneri, še verže šovra i riži e el brodo. Kužinar apjan šoto

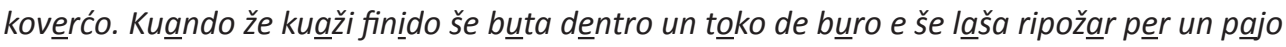
de minuti.

\subsubsection{PESCE}

Il pesce si mangiava quasi ogni giorno ed era il simbolo della cucina lussignana. Questo era molto dipendente dalla difficoltà di conservazione alla quale, fin da tempi remoti, gli isolani avevano cercato di provvedere in diversi modi. 


\section{Essicazione}

Il sistema più semplice era quello dell'essicazione. El folpo (polpo) ed el grongo erano quelli che si adattavano meglio a questa operazione: infilzati su un ramo di tsrnika (leccio), venivano esposti all'aria e al sole. Un altro sistema per conservarli era quello di šalarli e poi appenderli sopra il fuoco di piante odorose quali smrekk (ginepro), in modo che il fumo li asciugasse completamente. A questo punto, posti in un luogo fresco e asciutto, come la cantina, potevano conservarsi a lungo.

\section{Salatura}

Per le sardela, invece, la conservazione ottimale era la salatura. Alla fine di aprile o in settembre, quando la loro cattura era più abbondante, si ponevano in recipienti di legno, cosparse di sale tra uno strato e l'altro. Quando il recipiente era pieno, si versava sopra una salamoia, poi si poneva un coperchio in legno e su di esso un grosso sasso. Venivano lasciate così per sette mesi, dopo di che si potevano consumare.

\section{Conservazione sott'olio}

Per i pesci più pregiati come il rombo o il tonno, invece, si ricorreva al sistema della conservazione sott'olio. Dopo una leggera bollitura con acqua e aceto, il pesce veniva posto a grossi pezzi in vasi di vetro e coperto con ojo de olíva.

\section{Žaladja o ǵeladja}

Nel XVII e XVIII secolo era in uso un sistema, per conservare i pesci migliori come brantsinini, dentali, paǵei e orade, descritto dettagliatamente dal Nicolich: "Consisteva questa preparazione, conosciuta sotto il nome di žaládja o ǵeládja, nel far bollire il pesce in sufficiente quantità di olio puro, ed appena compiuta la bollitura, il pesce così cotto si stivava in barili per farne smercio. Un pesce [...] così preparato, dopo che era raffreddato, si poneva in un apposito recipiente, vi si gettavano sopra qualche grano di garofano, oppure un po' di zafferano, qualche foglia di rosmarino, od un po' di polvere di canella, ed era così sevito a tavola come oggetto di lusso..." (Nicolich 1871: 63).

\section{Brodeto}

L'antica sapienza ha consentito anche di produrre il brodeto, inventato per utilizzare tutte le qualità di pesce disponibili e soprattutto quelle ritenute povere, ma così ben amalgamate e combinate insieme da costruire un piatto squisito e nutriente. Tipico di Lussino era il brode to de grongo e škarpena.

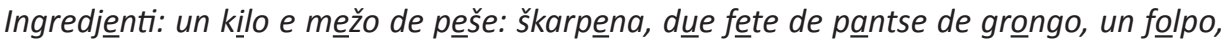
una šepa, un kalamar, due féte de tono freško, tsivola, ajo, peršemolo, farina, tsukero, atseto, konšerva de pomodoro, ojo de olivia

Preparatsjon: in una pińata še mete nêl ojo de olivva, meža tsivola intjera, ajo, peršémolo, e šubito anke el peše (prima el folpo a petsi, poj el kalamar e la šepa). A meta kotura še aǵunǵe mežo lịtro de akua dovve še ga škuaja dúe kućarini de farina, uno de tsukero, dúe de atsêto e méžo de konšerva. Kuando že kuaži finido še mête el rešto de peše tajado a fête, še šála e še kopre kon un poko de $\underline{a} k u a$.

\section{Savor de peše ala lušińana}

Ingredjẹnti: un killo de žgombri pịkoli, un killo de sardẹle o angužigole (anguglia) o

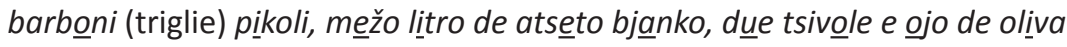


Preparatsjon: še puliše el pẹše, lo še infarina, friže, ti butti šal e še laša in una salvjeta. Še taja le tsivole a meža lúna e še frị̌ze túto apjan in ojo de olịva, še aǵunǵe el atsêto e el vin. Še ašpeta ke fa un bojo e še dištuga el fogo. Dentro še mête una parte del peše ke el štaga koži

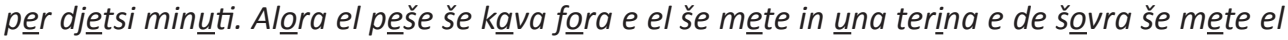
lavrano (alloro), pevere e la tsivola frịta. Denovo še škalda l atseto e el vin e še mête el pẹše ke že reštado. Kuando tutto el peše že in terina, de šovra ge še buta el šugetto, še kopre túto

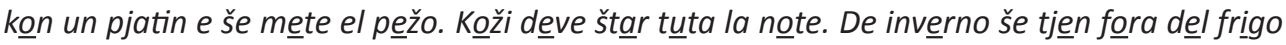
e dura tanti ǵorni.

\section{Barboni de Palazjol ${ }^{5}$}

I barbonni (triglie) più buoni erano quelli di scoglio e in particolare queli presi d'estate, perché sono più piccoli e più saporiti. C'è perfino un detto: Tešta de barbonn no že per el šervo, ma per el paron.

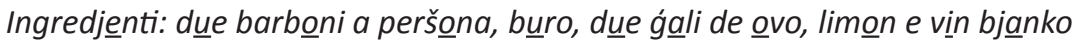

Preparatsjon: pulir bẹn i barboni , infarinarli, šalarli e frižerli in buro abondante. Lašăr ke še rafreda. Nel buro de kotura, ke še mete in un podić (pentolino), še aǵunǵe i ǵali de ovvo, šugo de limon e še kužina túto mišjando in kontinuo a bańomarịa pe venti minúti. Konži še fara una šalṣ̌̂ta. I barbonni še mete in una faršora, še kopre kon la šalša e še mete in forno per poki minuti.

\section{Sardele impanade}

Tipico piatto lussignano.

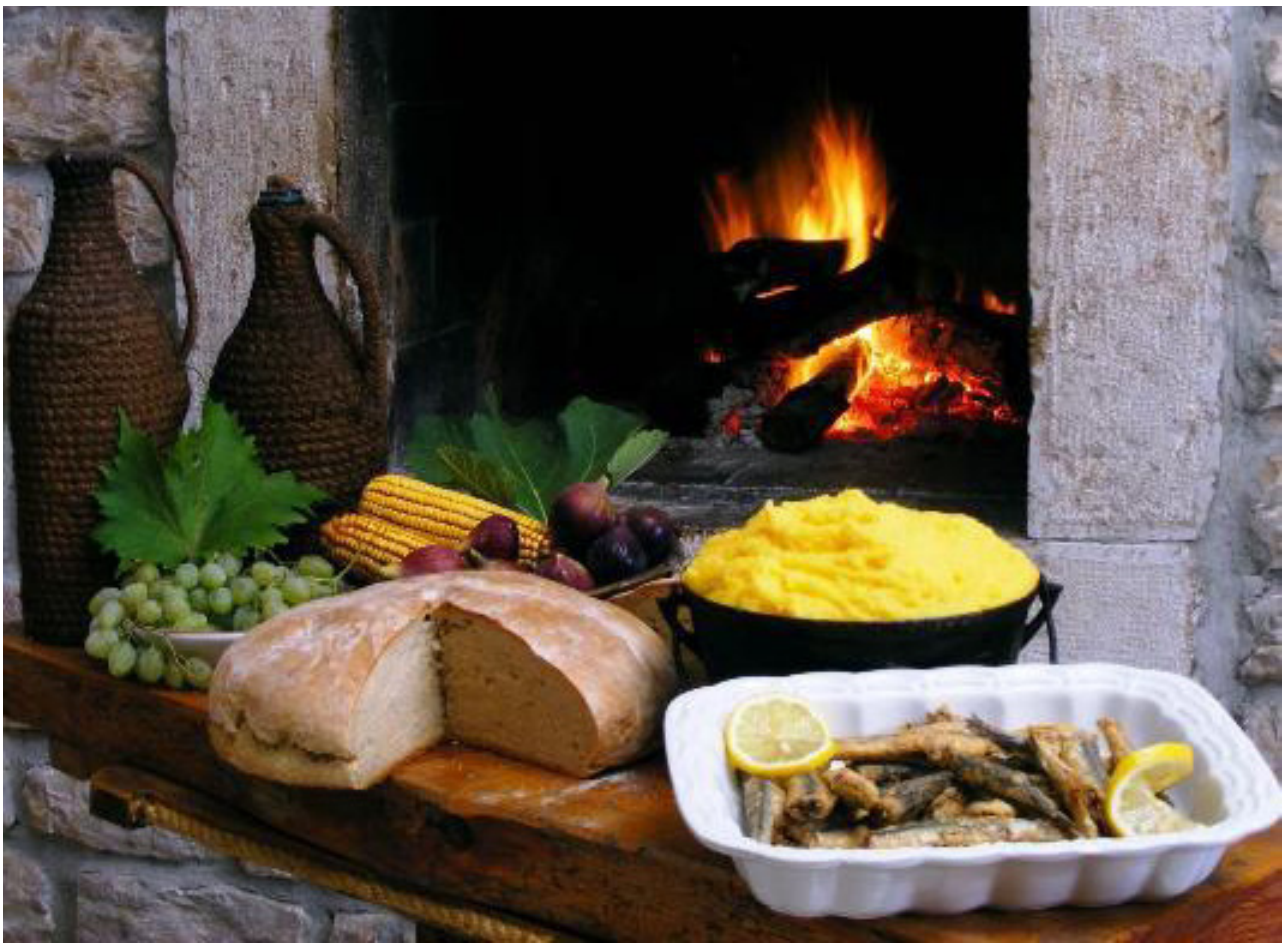

F. 3: Pietanze lussignane (http://www.tz-malilosinj.hr/Losinj.aspx?lzbornikld=71, 20.7.2010.) 


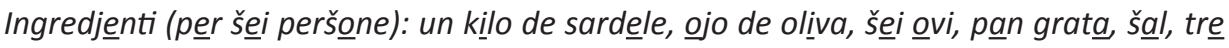
limoni

Preparatsjon: pulír le sardẹle, tajarge la tešsta e kavarge i špini, lavarle, šalarle e lašarle škorlar. Pašarle, prịma nella farina, poj nej ovi žbatudi e nel pan gratá b baterle kon un kortelo in mańera ke la panatura non še štakk. Mẹter šul fogo la faršora, frižerle e poj šugarle kon la salvjêta.

\section{Palamida in ojo}

Ingredjenti: due kili de palamide pulide (tajade a petsetini de tsirka dúe čentimetri),

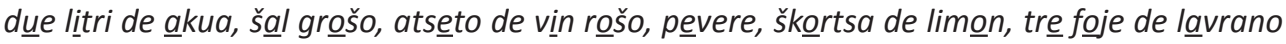
(alloro)

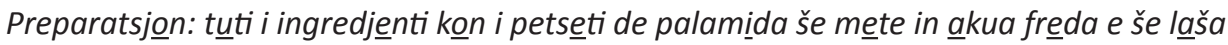

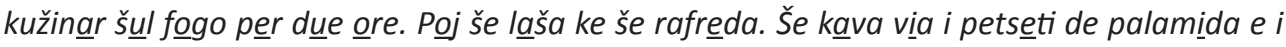
še láša škorlar durante la note. El šekondo gónorno i petsetini še devve pulir dála pêle, i špini e la parte škura. Koži pulídi i še mête in važeti, koži ke framežo ge rešta un póka de arja. Še verži dentro el ojo de olîva, pevere e un pajo de foji de lavvrano. Dopo šete ǵorni še pol mańar.

\section{Peše lešo}

Ingredjẹnti: un kịlo de peši: škarpenna, moli, brantsinini..., karota, peršêmolo, šéleno, ajo, tsivola, ojo de olivva, rị̌i, šall, pevere

Preparatsjon: še kužina el peše in akua kon peršemolo, ajo, šeleno tajadi in fin, la tsivola a metá, la karota, ojo de olivva e šál. Poj še kava vịa dẹla pińata el peše e še škorla el brodo. Še škjatsa la karota, šeleno, peršêmolo e tsivola e še buta dinovo tuto in brodo ke še kužina kon i riži. Še kondiše el pẹše kon ojo de olivva e še mańa.

\section{Peše in gradela}

El peše pjü bon jera kuel ke še fatsevva in gradella.

Il pesce fatto alla griglia aveva tutti i sapori perché il fuoco era alimentato di rami de tsrnika (leccio), rami de olíva (quando si puliva l'olivo non si buttavano i rami ma si portavano a casa) e di vida (vite).

Ingredjenti: pešse (barboni, sardela, menole ${ }^{6}$ ecc.), peršemolo, rạmi de ružmarinn, ajo, ojo de oliviva, šal

Preparatsjon: pulir el peše dạle šćame (squama) e la pantsa e bižońa mêterlo šule gradele kalde. Lašarlo roštir ben šu una e poj šul altra parte. Non dimentikar ke kuando una parte že roštida bẹn, alora še onže kon el ramêto de ružmarịn bańado in ojo. Kuando el pẹše že pronto še mête de šovra el ojo de olịva, peršêmolo e ajo tajáji in fin.

\section{Kalamari ripjẹni}

I calamari si prendono nella stagione fredda, da novembre a febbraio, poi spariscono e i pescatori dicono: El kalamaro že anda drijo la sardéla, infatti seguono le masse del pesce blu, soprattutto le sardine.

Ingredjenti: kuatro kalamari, ajo, pạn grata patate, peršemolo, ojo de olịva, šal, pevere

Preparatsjon: pulír i kalamari, kavarge i occi e i kukuriku (bocca), la tešsta še taja a petsetini. Še mišja i petsetini de kalamar kon el pan grata, peršemolo, ajo, ojo de olivva, šal

Maride - pesce simile alle menole. Siccome era destinato alle classi meno abbienti si usava anche dire : Le maride že le luganige déj lušińani. II mio bisnonno aveva il soprannome Menulić; ogni giorno per merenda prendeva la cistella e andava in Valdarke a pescare le menole; e da qui il soprannome. 
e pevere e še impińiše el kalamar. Še kjude kon el štekadente s $^{7}$ stecchino). Še mete in pińata un poko de ojo e še buta i kalamari ke un póko i še rožzola. Kuando i ga prežzo un bêl kolor

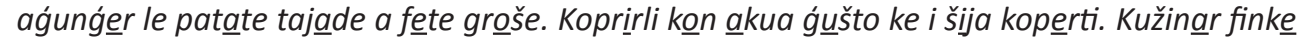
el kalamar non šara tenero. Provar kon el piron, še el páša alora že pronto.

\subsubsection{CARNI}

\section{Jelitići de kapreto}

Jelitići sono le trippe di capretto. I vecchi lussignani avevano l'usanza di andare a lavare le budella in mare cosicchè avevano un sapore speciale.

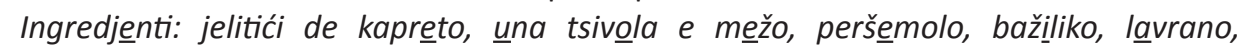
pomodori, patate, due kućari de ojo de olivia, šal e pevere

Preparatsjon: še fa rožolar šul ojo la tsivola tajada. Še taja i budej a petsetini de dú čentimetri e še láša frižer apjạn. De šovra še mête el peršemolo e šeleno. Še špela i pomodóri, $i$ še taja a petsetini, e še butta in pińáta, še aǵunǵe pevere, šal, lavvrano, un poko de akua tjẹpida e še laša kužinar apjan per un ora. Mišjar špešo. Le patate še taja a dadini e še buta dentro. Finke le patate non šara pronte, pero non le deve andár in papa (spappolarsi), bižonna kužinar apjạn tüto inšjeme.

\section{Kalandratsa o kalandrakka}

Kalandratsa o kalandraka era uno stufato di carne, patate e pomodoro. Ti že una bravva koga (cuoca) a far la kalandratsa.

Ecco la ricetta:

Ingredjenti: karne a šelta, mežo killo de patate, tre pomodori grandi, tre tsivole, dú špigi (spicchi) de ajo, tre kućari de ojo de olivva, peršemolo, bažiliko, šal, pevere

Preparatsjon: Tajar la karne a petsi grandi (non butar vịa i oši). Frižer per djêtsi minuti la karne e mišjar šempre. Žontar (aggiungere) i pomodori špeladi a petseti o un kućar de šăša de pomodoro. Kužinar a fogo lẹnto. Meter peršemolo, bažiliko, šal e pevere. Žontar ákua tjẹpida fino ke la karne non diventa tẹnera e le patate tajáade a dadịni. Kužinar ankora un poko finke non že pronto.

\section{Šalăta kon karne lẹša o oštuantsi de karne}

Oštuantsi sono i resti di carne, a Lussino non si buttava via niente.

Ingredjenti: karne leša, ovi, ojo de olivia, atseto, meža tsivola, olive, šal, pevere

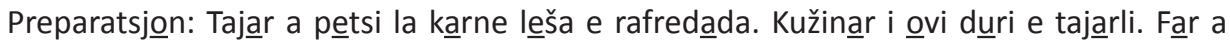
petsetini la tsivola, mišjar tưto in una terina e že pronto.

\section{Polpete}

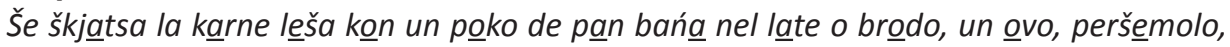
formaǵo, limon gratado. Še fa le balete ke še friže. 


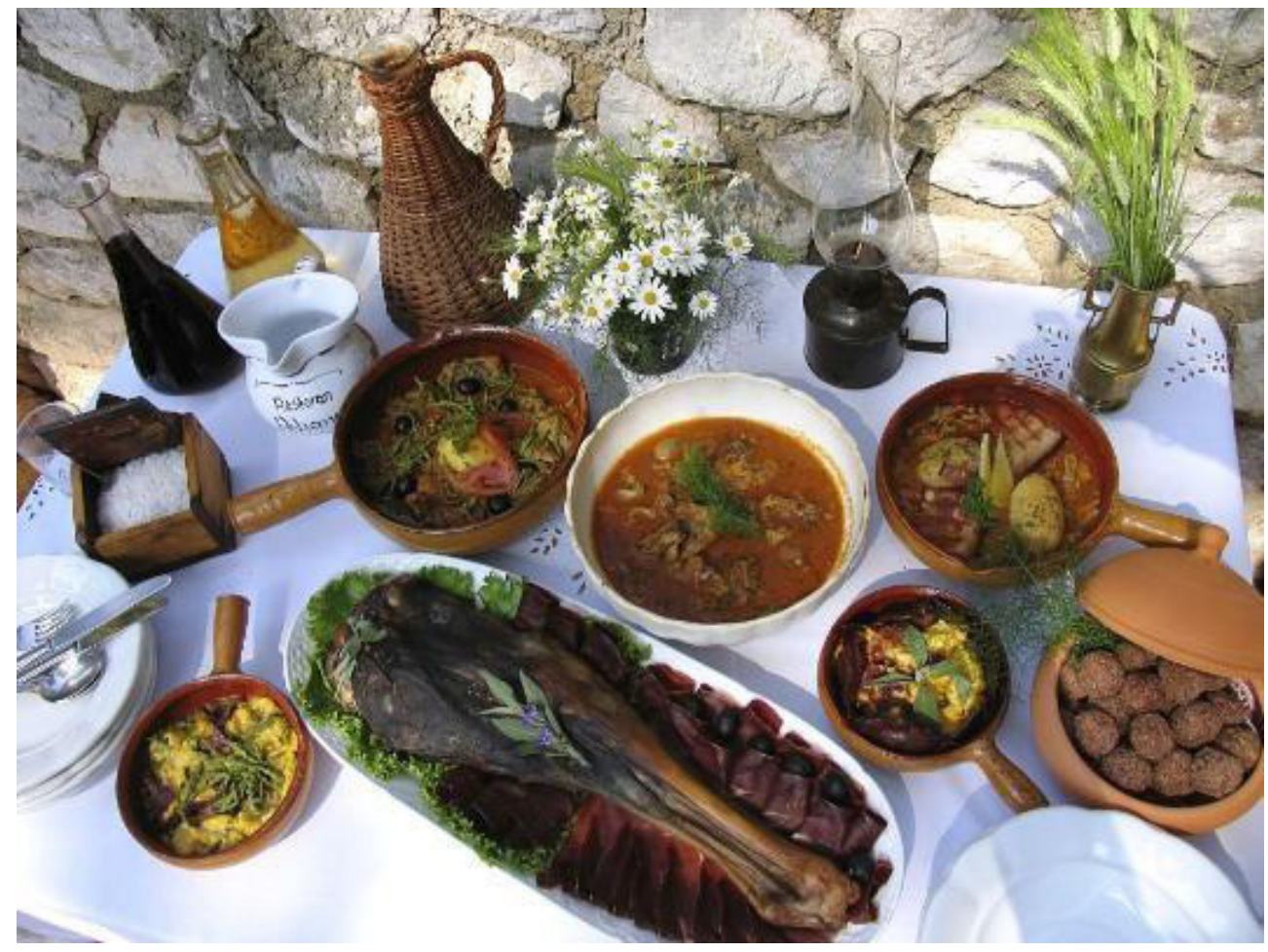

F. 4: Pietanze lussignane (foto V. Ivanović)

\section{Galina rošta}

Si mangiava quasi ogni domenica.

Ingredjenti: una galịna, šálvija (salvia), peršemolo, ojo de olịva, šal e pevere Preparatsjonn: še taja la galina in oto pẹtsi, še mête in una téća e še mête peršemolo, ojo e šál. Koprir la tẹća kon el koverćo dopo ke la galína ga ćapa un pọko de kolorr, e lašar ke še kužinina.

\subsubsection{DOLCI}

Quando la Natura incontra la Tradizione...nascono i doltsi della nonna.

I doltsi si preparavano soltanto per le feste. Raramente si facevano di domenica per fare felici i bambini.

\section{Doltse de čokoláta}

É un'antica ricetta di Lussino.

Ingredjenti: duetsento grami de buro, duetsento grami de tsukero, tsento grami de kakăo, tsento grami de biškoti šuti ${ }^{8}$ (secchi), ts $\underline{e} n t o$ grami de mandole, un ovo

Preparatsjonn: kužinar el buro e žbaterlo ben kon el tsukkero, aǵunǵer el kaká, i biškoti šuti, le mandole mažinade e el ovvo. Žvodar el impašto in un štampo, ke prima še ga imbura, e meterlo in frigo per oto ore. 


\section{Budin lušinan de gris}

Ingredjenti: šetanta grami de gris (semolino), šetanta grami de tsukero, un kućaro de buro, mežo litro de late, uvête, due ovi, škortsa de limon

Preparatsjon: veršar el grịs in late ke boje e kužinar per djêtsi minuti. Aǵunǵer el buro, un ninin de šal e tsukero. Še mete via del fogo e kuando el gris že tjepido še mête dentro $i$ ǵáli de ôvo, la uveta, la škortsa de limon. Dẹl bjanko de ovvo še fa la nevve e še aǵunǵe déntro. Še mete in un štampo kon tsukkero karameláto e še kužina a bańomaria per kuinditsi minuti.

\section{Pašamête de oržo}

Pašamête (fette biscottate) jẹra el pan non rošto fino la fine e tajáado a fetine.

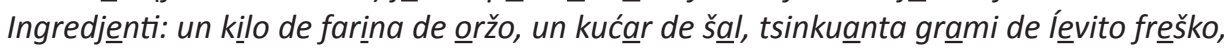
un poko de ákua tjepida, un kućar de tsukero

Preparatsjon: mišjar el ĺevito kon tre kućari de farina, akua tjepida kuanto bašta e tsukkero e lašar ke še altsa. Aǵunǵer al levito i altri ingredjenti e lašar dinovo ke še altsa. Še

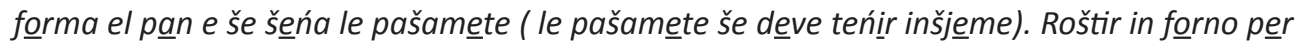
un ora. Poj še štaka le fête e še mete dinovo in forno ke le še šugga. Bańar le pašamête kon el vin o intsupar kon el a kua e mańar.

\subsection{BEVANDE TRADIZIONALI}

\section{Akuavita}

Si conservava dopo la distillazione delle vinacce per diversi anni. Si usava offrire da bere agli ospiti un bićerin, e si usava anche bere per mejo digerír.

\section{Bevanda lunga}

Pju $\underline{a}$ kua ke vinn. Sia uomini che donne usavano bere il vino diluito in acqua. Si beveva più vino rosso che bianco.

\section{Kafe de oržo}

Il caffè di orzo abbrustolito si serviva alla mattina ai bambini per colazione. Qualche volta si beveva anche alla sera.

\section{Kamomila}

Quasi tutti avevano la camomilla nel proprio giardino e facevano il tè.

\section{Sopa de vin}

Si usava bere d'inverno.

\section{Tè de šambuko}

Oltre a bere il tè, i vecchi con i fiori facevano i suffumigi per il mal di testa.

\section{CIBI PER I GIORNI FESTIVI}

\subsection{Feste religiose}

Ogni 13 dicembre la veneta Santa Lucia recava figi, nože (noci) e dolci ai bambini lussignani fino alla prima guerra mondiale, mentre San Nicolò più generoso portava anche giocattoli. Ma più munifico ancora arrivò Gesù Bambino e a quel tempo l'albero di Natale cominciò a far capolino in tutte le case. 


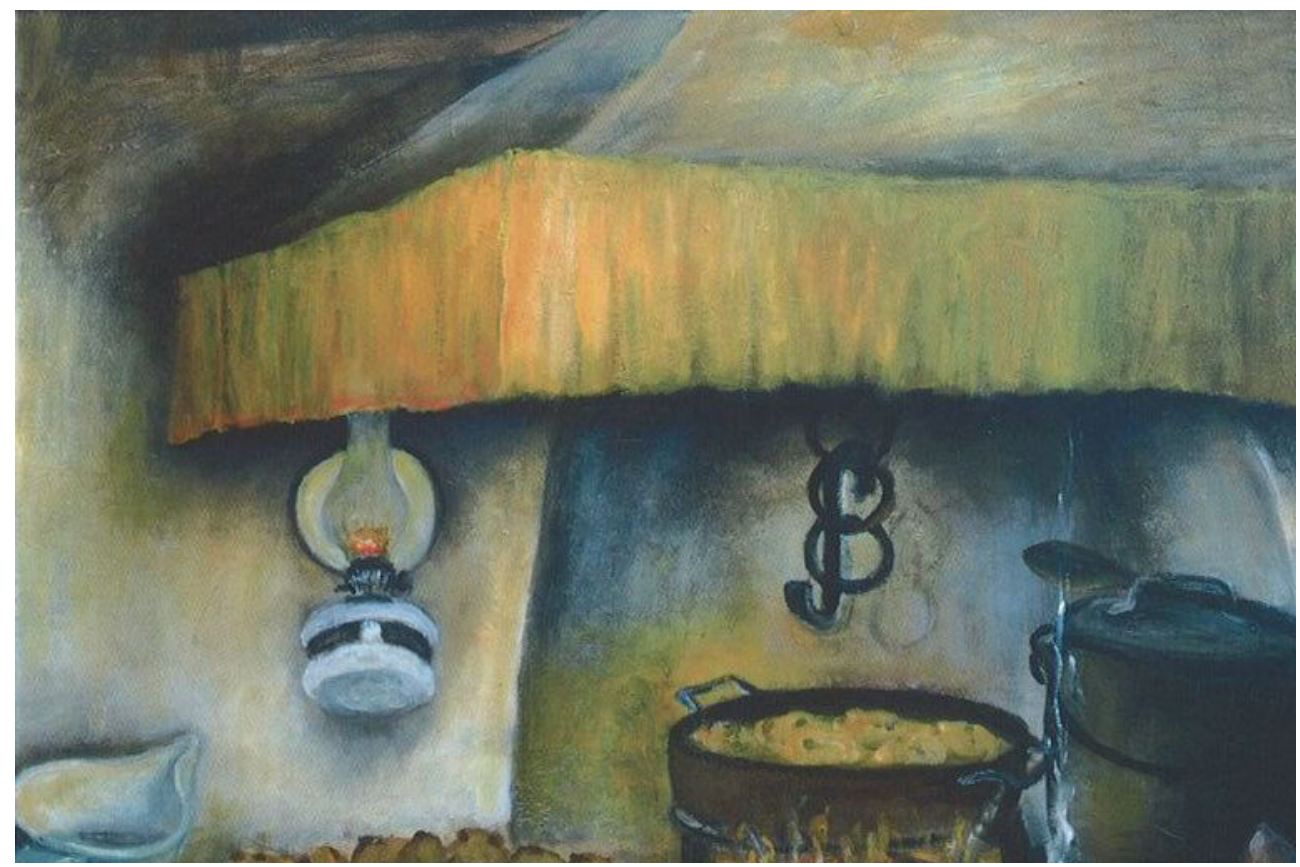

F.5: Piatti tipici natalizi (Vigilia di Natale, pittore lussignano Mario Glavan)

I Nadái a Lussino si susseguirono senza cambiamenti fino alla seconda guerra mondiale. Di nuovo la fame, bombardamenti, tedeschi, slavi e alla fine Gesù Bambino divenne Djed Mraz (Nonno Inverno).

Nonostante i tragici eventi, la forza dello spirito di economia e di ingegno degli abitanti di Lussino che sfruttando le magre risorse del suolo e le ricchezze del mare, riuscirono a creare una tradizione nella confezione di pochi, solidi piatti preparati e offerti con senso di sicurezza e superiorità. La padrona di casa era una donna pratica. Si liberava degli uomini che invadevano la sua area. Mandava il marito a "cior" in campagna l'albero. "Cior" è la parola giusta perché molto ma molto di rado si vedeva a Lussino un albero di Natale comperato. Era stato sempre rubato nella campagna degli altri. Così lei aveva tempo per preparare le specialità natalizie.

\section{Bakala}

Il bakalà si mangiava alla vigilia di Natale.

Ingredjenti: mežzo kịlo de bakala šuto, kuatro špigi de ajo, kuattro kućari de ojo de olíva, šal, pevere

Preparatsjon: el bakala še metevva a mojo per tre ǵorni e ońi ǵorno ge še kambjáva I akua. Še pulivva, tajavva a petsi e škjatsavva kon el piron. Še meteva ajo, pevere, ojo de olivva e še mišjava ben.

\section{Verže na pofrih ${ }^{9}$}

Per Nadal ti fa šempre le verže na pofrịh? Šikuro ke ši...ma ti la fa de kućaro o de piron? Ognuno col suo metodo era convinto di fare la verže migliore

9 Questa specialità chimavano anche Broskva na pofrịh. 
Ritseta: meti ojo šufičente šekondo la kualita ke ti fara, pjutošto un poko de pjü ke de meno. Poj in ojo fredo mêti un toketin de kalamaro, še el že pịkolo ti lo pol meter tuto, še no ti lo taji in kuạtro pêtsi, una rappa de kuele doltsi anke tająada in tre o kuatro petsi. Tuto ti mêti šul fogo per šofrižzer un póko, ma non tropo kožị ke non te čapa per la gola. Fâto kuešto, ti meti due litri de akkua freda o kalda, indiferente. Kuanndo komintsja bojịir, ti meti un poko de erbete, verže, un kapuntso, un pọko de panókja, patạte kuante ke ti vol pero intjere, šal kuanto bạšta e lašsa tụto kužinạr inšjẹme.

Kon la patsjentsa neta el kalamarr, apjạn, apjạn levi ge la tešsta kon i teštikoli e ti trovera la petuja, unna boršeta bjạnka, ti la mêti in una čikareta, e kuạndo la verrže že a mêta kotura, ti ge da unna mišjąada kon šta petuja e el brodo diventa bjạnko.

Fa un brodêto de pešse, kualšijąši peše, e kuel brodo de brudêto kuanndo kavi la verže per portarla in tâvola butilo šovra.

Mangiar da orbi!

\section{Prapańaki}

Venivano dai Balcani ma furono subito adottati grazie alla loro composizione fatta di olio d'oliva, zafferano e miele, tutti prodotti dell'isola. Si preparavano molto prima di Natale.

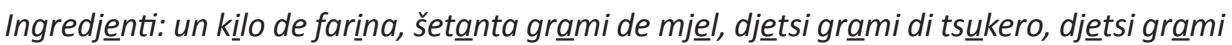
de mandole, ojo de olivia, škortsa de limon e žaferano

Preparatsjon: kol mjel e ojo še škota la farina mišjando kon la mutvica (mestolo) finke la pašta non diventa lišsja. Še aǵunǵe el rešto. El žaferano in polvere še mišsja kon un póko de vinn.

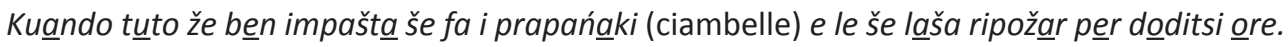
Prịma de mêter in forno še devve fár pikoli šenjẹti dekorativi kon el piron. Dopo, ankora kálde de forno, ki ke gaveva le man ferme le dekorava kon el tsukero e bjanko de ovvo.

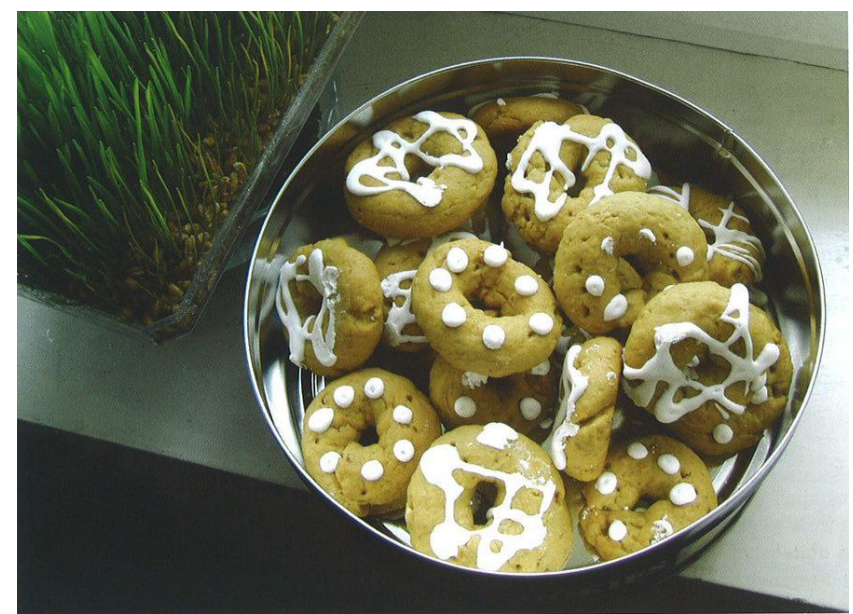

F. 6: Prapańaki (Nicolich 2007: 56)

Gli isolani iniziavano a mangiare le ciambelle dalla vigilia di Natale in poi e servivano anche come doni di Natale per parenti e amici. 
Fritole (Bragato 2007: 313)

Molto più emozionante era la preparazione delle fritole. La sera dell'antivigilia, fatto scendere il malva $s^{10}$ dal soffitto per preparare la pasta delle fritole.

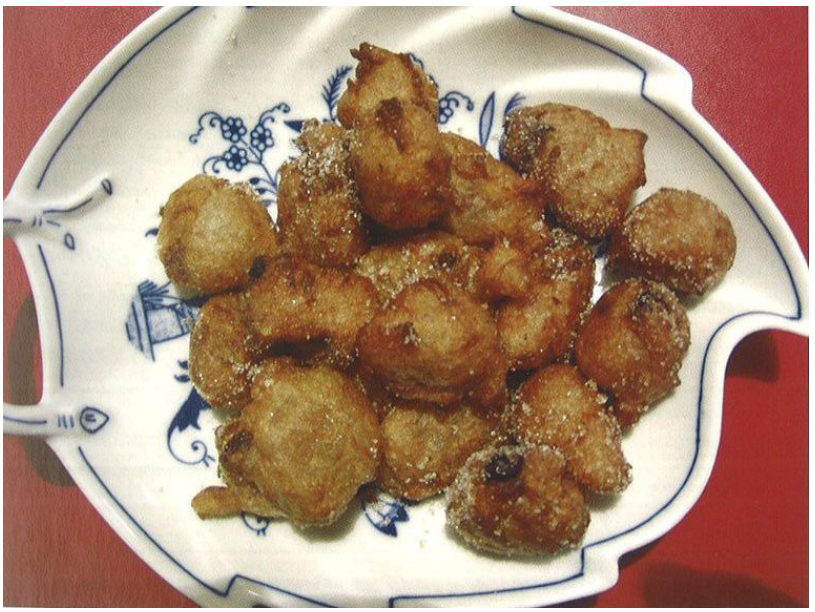

F. 7: Le fritole (Nikolić 2007: 43)

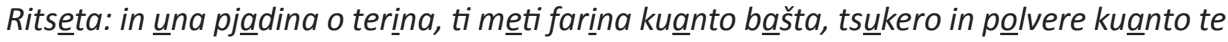

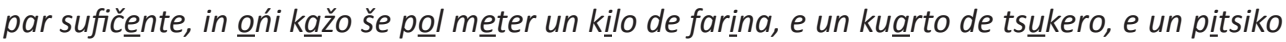

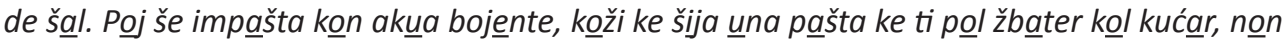
dura, non tenera, ti ke da kuatro bone žbatudde, poj ti aǵunǵi dú bićerini de rúm e ti žbáti dinovo per meža ora. Ti ge meti i ovvi, pińoj, limon e narantsa gratádi, un pokko de garofano

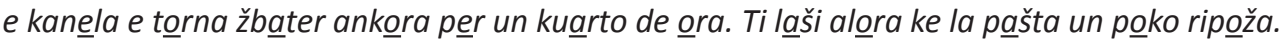
Alora ti le pol frižžr. Non šta mêter tropi ingredjenti, una koža ǵušto de túto póko. Molte volte

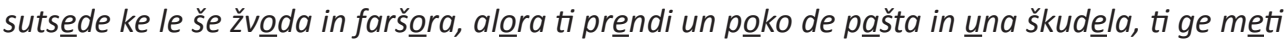
la farina e ti mišji e žbati benen. Še poj le že dure e non le še dištaka dála faršora, alora provva in una škudela meter un poko de rüm, e ti vedera ke te šara mejo.

Al mattino della vigilia la padrona di casa cominciava a friggere le prime fritole per merenda.

\section{Krakavice}

I bambini per Natale, trovavano sotto il cuscino le krakavice e alcuni fichi secchi.

Eko la ritsêta: un killo de farina, tsinkuanta grami de ĺevito, un kućar de šal. Še mišjava el ĺevito kon due kućari de farina e akua tjepida e še lašava ke lévita. Al impašto ĺevitado še aǵunǵevva farịna, šal e a kua. Še lašavva ke ĺevita ankora. Še formava kon la pášta kome un tubeto de tre tsentimetri de grošetsa e lungi kuaranta. Še univa le tsime e ge še dáva una forma ovale.

\section{Pan de figi}

Pan de figi era un dolce di fichi secchi che si preparava nel mese di settembre e si mangiava alla vigilia di Natale.

Ingredjenti: figi šuti, uveta, garofano, grapa

10 Il malvàs è un recipiente di legno o di terracotta a forma di un'enorme ciotola talvolta fornita di un manico come un pentolone gigante. 
Preparatsjon: še mažina i figi šuti. Še aǵunǵe i garofani. De što impašto še fa unna forma de piramide e še mete la grapa.

Si serviva a forma di rondelle cominciando a tagliare dalla punta.

\section{PASQUA}

"Via tutti dalla cucina!"- grida la mamma. Escono per ordine il gatto, il cane, il marito, i figli. La mamma si accinge a preparare le pintse (Bragato 2007: 269).

\section{Pintse}

Questa è la regola: prima si impastano, poi, per due volte si domano (si lavorano con le mani) e infine per tre volte le pinze si levano (lievitano).

Ingredjenti (per due pintse): un killo de farina, tretsento grami di tsukero, tsinkue ovi (kuatro per la pašta e uno per špalmár la pintsa), un póko de late, la škortsa de limon e narantsa, tsento grami de buro, tsento grami de ĺevito, un kućarịn de šal

Preparatsjon: še mišja el ĺevito, un pọo de late, una čikeritsa de kafe de farina, un kućarin de tsukero e še láša ke lévita. Še mišja kon la mutvitsa la farina, tsukero, buro (škaldado a

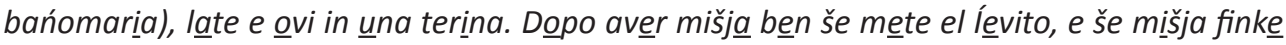
non še otjen un impašto morbido; še že nečešario še žonta un poko de late tjepido. Še kopre la pašta kon la kanavatsa (canovaccio) e še laša ke ĺvita. Poj še lavora la pašta žontando la

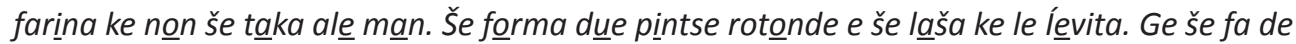
šovra una krotse e še špalma kon un ovo žbatudo. Še rošte in forno de leníi.

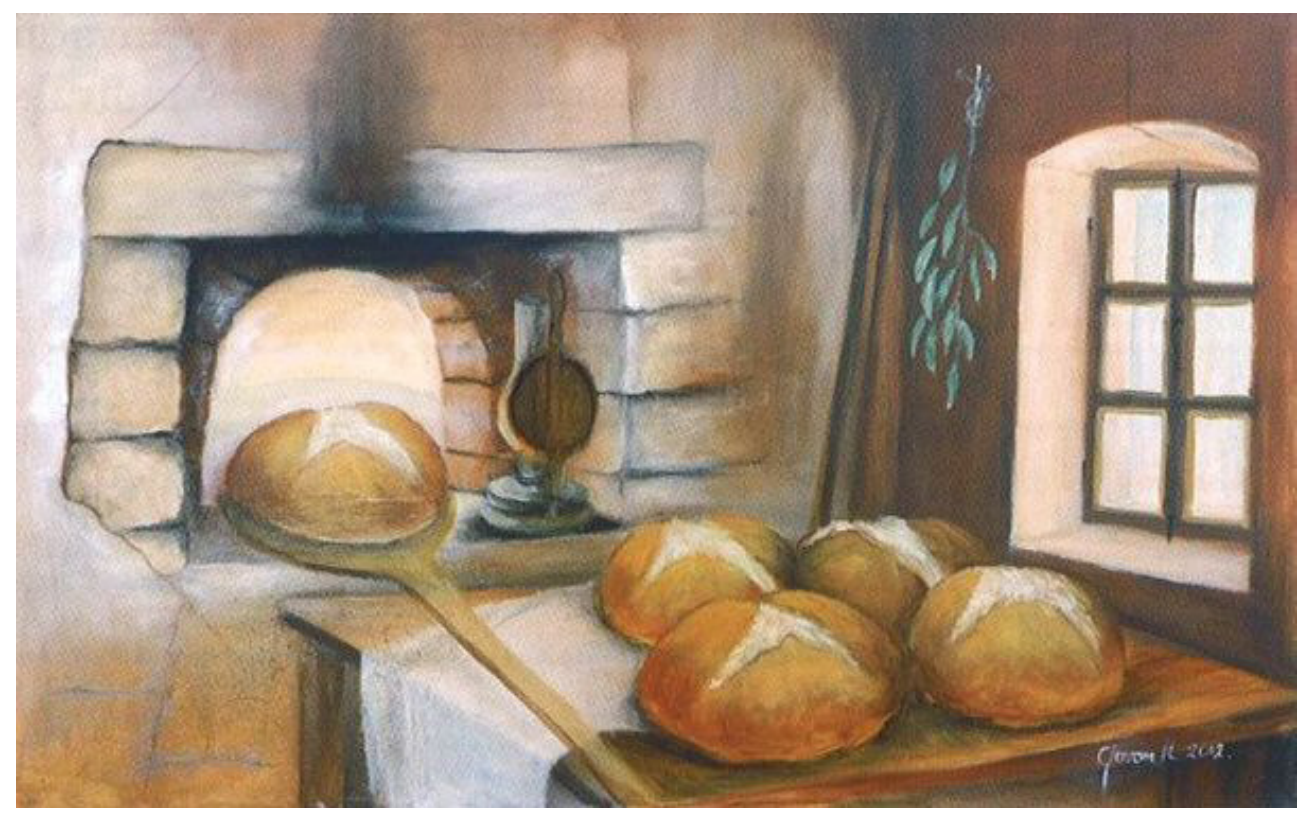

F. 8: Le pintse (Nikolić 2007: 81) 


\section{CARNEVALE}

I tipici dolci di carnevale erano l'olito e i galani.

\section{Olito}

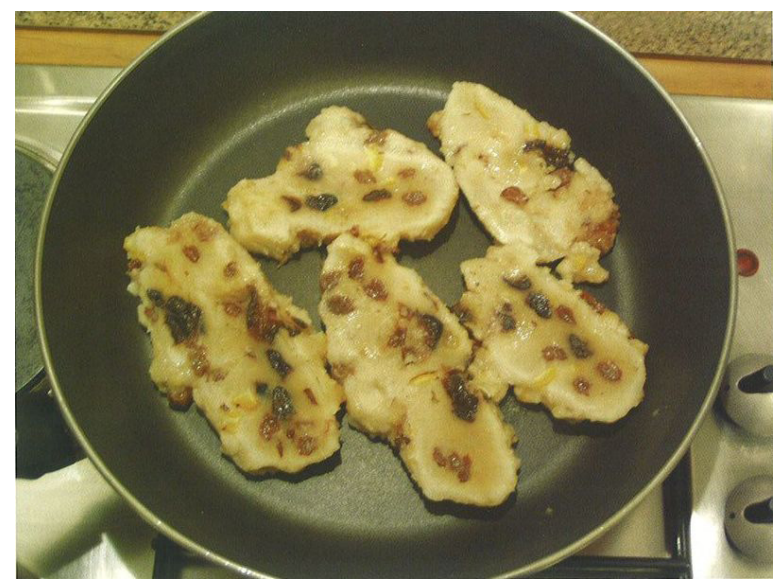

F. 9: Olito (Nikolić 2007: 42)

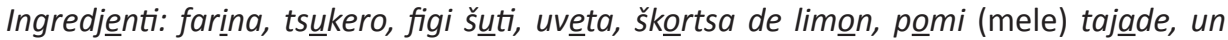
poko de ojo, šal e late

Preparatsjon: še mišjavva túti i ingredjenti in una pạšta e ki ke gavevva, podevva meter anke el lardo de pékora. Kuešsto še metevva in štomigo rovešado della pêkora. Še kuživa i orli

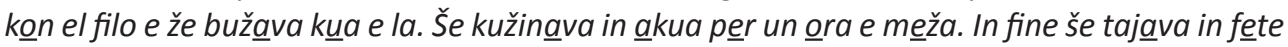
large un tsentimetro e še šerviva kaldo, fredo o anke škaldạdo šul ojo.

\section{Galani}

Aj ultimi de karneval še mańa i galani (crostoli). I galani še fa kon farina, ovi, tsukero, škortsa de limon; še fa el švojo, še taja in tante štrike, še li ingropa e še li frị̌ze in ojo.

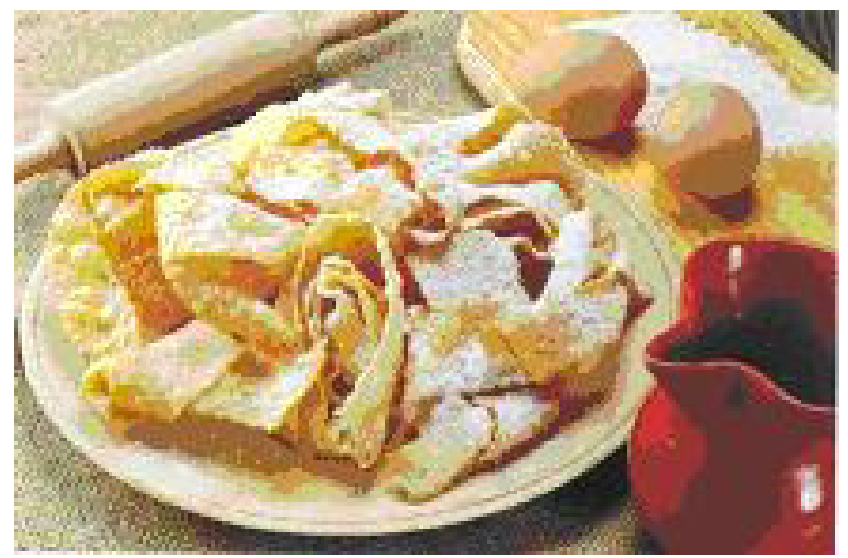

F. 10: I galani (http://www.gastro.hr/Krostule-s-vinom-1741.aspx, 20.7.2010.) 


\section{GIORNO DEI MORTI}

Per il giorno che si ricordano i morti, il 2 settembre, a Lussino c'era l'usanza di fare questo tipico dolce:

\section{Fave lušináne dej morti}

Ingredjenti: duetsento grami de mandole, tsento grami de tsukero, tsento grami de farina, trenta grami de buro, un ovo, kanela, škortsa gratáda de limon

Preparatsjon: še špela e mažina le mandole, še mišja kon el tsukero, un ovvo, škortsa de limon e un ninin de šal. Še forma la pašta morbida e aǵunǵe un pajo de goče de grapa. Še forma kome dele pịkole fáve, le še mête in un lamarin imburado e de šovra le še špalma kon el ǵálo de ovo žbatudo. Še kužina in forno per venti minuti tsirka.

\subsection{Feste familiari}

\section{Krokante}

Per festeggiare gli sposi, dopo la cerimonia in chiesa, era tradizione offrire agli ospiti il croccante che gli sposi rompevano tra gli applausi e gli "evviva" dei presenti.

Ritseta: pulír tretsento grami de mandole, tajarle a pikoli petsetini e meterle in un lamarịn. Le še deve roštir in forno finke non le čapa un póko de kolor. Intanto, še mête in un pińáta tretsento grami de tsukkero e še kužina šentsa mišjar finke non diventa róšo karamelato. In što tsukero še buta la mandole e un pajo de goče de limon. Še mišja túto bên kon la mutvica e še mete in štampi ke prima še ga onto kon ojo de mandole. Kuando še ga rafreda še kavva fora el krokante ke že diventa kọme vetro. Že onže ben un kortel kon ojo de mandola e še taja a petsi kuadrati, triangolari... Dopo $i$

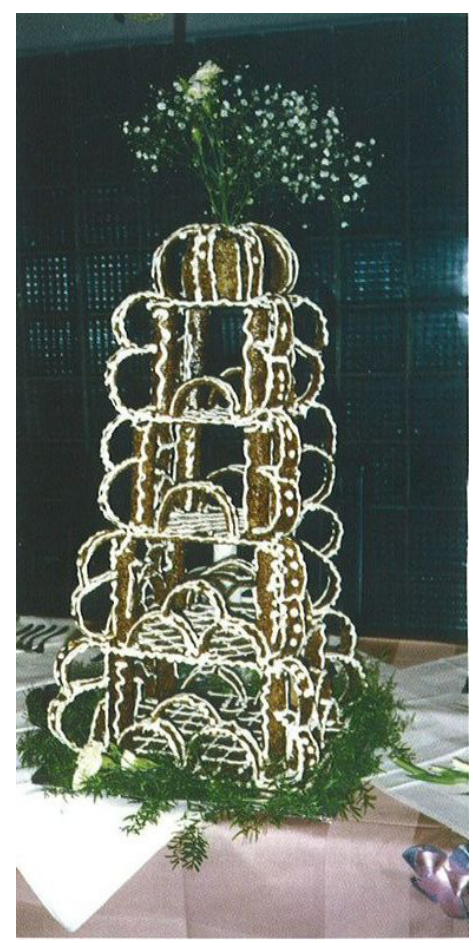
še táka kon tsukkero karameliža in figure ke še vol. Še fa la neve kon un poko de tsukero e bjanko de ovo e li še dekora. (Nikolić 2007: 53) 


\subsection{Momenti particolari}

Durante l'allatamento, le donne nei primi giorni dovevano mangiare dei cibi scelti. I bambini venivano allattati con il latte materno per molti mesi e pertanto l'alimentazione doveva esssere molto energica.

\section{Panada}

Ritseta: in una pińata de a kua freda še mete dentro bańăr le pašamete šfregolạde, un póko de ajo, ojo de olịva, šal e devve štar kőži fermo per venti minuti o mežza ora ke še intsomba ben el pan de akua. Alora še meteva bojiri, e pju tempo ke boje, pju bona vjen la panáda. Še la že trọpo fiša ti aǵunǵi ońi tanto un poko de a kua e ki ke gaveva formaǵo lo grâta de šovra. I lušińanni gaveva poko formaǵo perke el formáǵo i vendeva e el pan i mańava.

In caso di malattie si consumavano dei cibi leggeri. Le persone malate e deboli mangiavano brodo, soprattutto el kordjâl, così i lussignani chiamavano il brodo di gallina ristretto, cioé tanta carne e poco brodo.

Per i raffreddori si beveva il vin brule. Il vino si beveva caldo e si dava anche ai bambini. Si sentiva la mamma gridare: Šufite kol fatsoleto, non šta tirar el nažo, impara la kreantsa!

Ritseta per ešer feliči. L'arte della cucina è dentro le donne lussignane, e lo conferma un foglietto trovato nei quaderni ereditati:

Prendi un killo de kuor kontento, un kịlo de amor de lavoro, un matsolịn di modestia, un ramêto de rašeńatsjọn. Uniši tüto ben ben, meti in una pińata dela bona volontă, aǵunǵi...

L'elenco continua ma gli ingredienti sono, oggi, scomparsi; come le domestiche, come le maride (Bragato 2007: 214).

\section{FILASTROCCHE, MODI DI DIRE E INDOVINELLI}

A Lussinpiccolo le notizie si tramandavano da cucina a cucina, da strada a strada, e tutti venivano a saperlo.

Filastrocca che si sentiva spesso dire in cucina:

Pjovva, pjovižina,

la gata va in kužina, la rompe le škudele, la ląša le pjü bele, la kore šoto el letto, la trova un konfeto, el konfeto jera durro, la lo buta žo dẹl muro.

Canzoncina della cisterna:

La tsisterna de Lušin ${ }^{11}$

Kuando ti guardi el rubineto del a akua korente ma dịme: la tsisterna de Lušin, non te vjen in mente?

11 Scritta da Lina Gherbaz. 
Onni kaža ge ne gaveva una a Lušin

e špešo una altra, fora, per bańar el orto e el gardinn.

Akua ke dạl tsiel ge kaška al lušińan per ńente!

Per bever, e lavar, kužinar, tuto gratuitamente!

Glorja aj čikuti! Ke ne ga prešervạ da tanti guaj, difati, bevendo kuel akkua nišun že morto maj.

Ma...pjọve, non pjọve, drio el Kalvarijo ${ }^{12}$ že škuro...

"kuei že lampi de kâldo" - "Non pjovera de šikuro!"

In lúlo non pjovevva, la tsisterna še žvodạva:

alora la čeža una protsešjon organizava.

Atorno el Duomo, devôti tụti kuanti,

in fila per due še kantạva le Litanijie dẹ Šannti.

Kol ekuinotsijo de autunno, la tsisterna trabokava...

"Ápri el športel dela gôrla, ke I akkua in štrada vạda!"

Kon la pompa e kol šećo I akkua šu še tirava:

ma malora! Ke pegola! Še el gropo še molavva.

Aj! Aj! Mạma! In tsisterna me že kaškādo el šéćo...

Bavillo! A dịr ke bel jera, anke še el jera većo.

Komprạr un šećo non jẹra vera škalonna:

un pokko per la špeža...e i, štuanǵe, ke vergonńa!

Ke gavemo peršo el šećo, non štege a nišun kontạr,

Ke la Maria o la Ana ne podarija kritikar.

Invêtse kori prešto šu dạl šińor Giovanin

E dige ke el ne imprešti el rampin.

Prova, riprova...eko, me par ke lo go urta...

El že in pjedi o tombado? - Tăži...lo go inkotsa

Racconto scherzoso, realmente accaduto:

- Diceva la signora Giacomina: "Štašera me šon fáta in faršoreta un bel ovvo frešsko e me

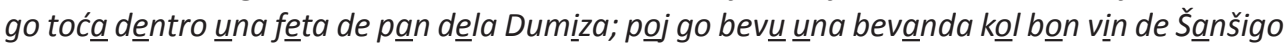
e I akua frešḱ dêla mía tsisterna e me šento proprjo benn!"

- "...e lei, signora Catina?" - "Ah mi go mańa un jajić kon un toketo de pan e una bevuandiza!" 


\section{CONCLUSIONE}

Vari fattori come la struttura del nucleo familiare e le condizioni di vita precarie della gente lussignana, che si basava sulle proprie risorse e il duro lavoro, hanno dato un notevole contributo alla nascita di piatti rustici preparati semplicemente. Ma semplicità, come si può pensare, non è indice di banalità o negatività, anzi significa genuinità e vivere sano.

La cucina lussignana, come si è visto, vanta alcuni piatti di una certa originalità, ormai poco noti, sbiaditi col tempo e divenuti piuttosto rari oggi, sebbene siano stati comunissimi in un tempo passato ma non troppo lontano. Ogni festività era collegata ad una particolare pietanza e questa era una delle rare occasioni per poter mangiare in abbondanza cibi conditi con tranquillità d'animo, reciproco affetto e benedizione. Ma non solo, nei giorni festivi I'intera famiglia si riuniva attorno al fogoler e passava piacevoli momenti raccontandosi aneddoti e storie di ogni giorno nella parlata tipica.

Al giorno d'oggi tali momenti si diluiscono sempre di più, ed il nucleo familiare non può più definirsi solido come una volta, quando ancora esistevano valori genuini.

Tornando al fogoler , questo era una volta il fulcro della cucina, e con esso molti altri accessori o altri oggetti che si usavano giornalmente e che ora trovano posto nelle soffitte e nelle cantine o più semplicemente sono andati persi.

"Le usanze portate con noi lentamente si perdono; sbiadiscono come le immagini dei nonni e bisnonni nelle vecchie fotografie. Ma, in loro difesa, sorgono i sogni." (Bragato 2007: 458).

\section{RIFERIMENTI BIBLIOGRAFICI}

\section{ABRAMIĆ 1975}

Arlen Abramić, Lošinjski talijanski govor, Mali Lošinj 1975.

\section{AUTORE IGNOTO 1921}

Autore ignoto, Ricette lussignane del 1921.

\section{BARTHES 1966}

Roland Barthes, Elementi di semiologia, Einaudi, Torino 1966.

\section{BRAGATO 2007}

Elsa Bragato, Una volta, a Lussin..., Comunità di Lussinpiccolo, Trieste 2007.

\section{DEVOTO - GIACOMELLI 1975}

Giacomo Devoto - Gabriella Giacomelli, I dialetti delle regioni d'Italia, Sansoni, Firenze 1975.

\section{DEANOVIĆ 1969}

Mirko Deanović, "Intorno alla nomenclatura peschereccia di Lussinpiccolo", in: Studi linguistici in onore di Vittore Pisani, Pandeia, Brescia 1969., p. 255-265.

\section{DEANOVIĆ - JERNEJ 2002}

Mirko Deanović - Josip Jernej, Vocabolario italiano-croato, Školska knjiga, Zagreb 2002.

\section{FAZLIĆ 2003}

Marta Fazlić, Nonina otočna kuharica, Profil, Zagreb 2003.

\section{FUČIĆ 1990}

Branko Fučić, Apsyrtides: kulturno-povijesni putopis po otočju Cresa i Lošinja, Narodno sveučilište, Mali Lošinj 1990.

\section{GASPAROTTO 1984}

Anna Chiara Gasparotto, Pescatori veneti di Lussino, Cierre Edizioni, Canova 1984.

\section{HIRC 2003}

Dragutin Hirc, Sulle isole Quarnerine, Pino Tuftan, Rijeka 2003.

\section{HREGLICH MERCANTI 1999}

Neera Hreglich Mercanti, Ricordando Lussino, [s.l.], Hreglich Mercanti, 1999. 


\section{LUSSINO 1999-2010}

Rivista Lussino, Foglio della Comunità di Lussinpiccolo, 1-33, aprile 1999. - agosto 2010.

\section{MAVROVIĆ 1994}

Nadir Mavrović, Cres i Lošinj: šetnja po otocima, otočićima i hridima, Art Studio Azinović, Zagreb 1994.

\section{MELLONE 2001}

Adriano Mellone, Ricette per un anno, La Fenice, Treviso 2001.

\section{MILIĆ 2002}

Carlo Milić, "Peccati di gola dimenticati: storie in cucina a Trieste", in Istria e Dalmazia, Edizioni Italo Svevo, Trieste 2002.

\section{NICOLICH 1871}

Mattes Nicolich, Storia documentata dei Lussini, Tipo.-litog. Istriana di Antonio Coana, Rovigno 1871.

\section{NIKOLIĆ 2002}

Adrijano Nikolić, Kuharski izazovi s moga otoka, ADI, Mali Lošinj 2002.

\section{NIKOLIĆ 2007}

Adrijano Nikolić, Otočna korabljica: kuharski zapisi, vl. izd., Mali Lošinj 2007.

\section{PITTANO 1993}

Giuseppe Pittano, Sinonimi e contrari: dizionario fraseologico delle parole equivalenti, analoghe e contrarie, Zanichelli, Bologna 1993.

\section{ROSAMANI 1999}

Enrico Rosamani, Vocabolario Giuliano: dei dialetti parlati nella Venezia Giulia, in Istria, in Dalmazia, a Grado e nel Monfalcone, LINT, Trieste 1999.

\section{SOKOLIĆ 2003}

Julijano Sokolić, Lošinj: storia, cultura, arte, bellezze naturali, Turistička naklada, Zagreb 2003.

\section{TRETJAK 2003}

Donatella Tretjak, Istria, Cherso, Lussino, Bruno Fachin Editore, Trieste 2003.

\section{VONDERWEID}

lolanda de Vonderweid, Ricette triestine, istriane, dalmate: antiche e moderne, LINT, Trieste 1997.

\section{ZINGARELLI 2004}

Nicola Zingarelli, Lo Zingarelli 2004: vocabolario della lingua italiana, Zanichelli, Bologna 2004.

\section{RIASSUNTO}

Il dialettto lussignano fa parte del dialetto veneto come conseguenza del lungo dominio della Repubblica di Venezia sul territorio dell'Istria e del Quarnero. Questo dialetto è parlato solo, come dicono i lussignani, da coloro che da generazioni hanno sempre vissuto sull'isola e si sta estinguendo purtroppo insieme a loro. Le nuove generazioni parlano il lussignano solamente nella famiglia di origine. A volte capita che lo utilizzino per comunicare con i nonni, che rappresentano l'ultima generazione ancora legata al passato e che cerca di conservare, tramite la lingua, la propria identità culturale. La cucina dei vecchi lussignani assieme alle altre tradizioni, ha orgogliosamente mantenuto la propria individualità e trasmette inconsciamente lingua, usi e costumi di una volta. 


\section{SAŽETAK}

\section{Kuhinja starih Lošinjana ogledana u njihovom govoru}

Lošinjski dijalekt dio je mletačkog dijalekta i posljedica je duge venecijanske vladavine na području Istre i Kvarnera.

Ovim dijalektom govore, kako kažu Lošinjani, samo oni koji već generacijama žive na otoku i on, nažalost, nestaje s njima. Nove generacije poznaju lošinjski dijalekt isključivo ako je u njihovom obiteljskom naslijeđu. Ponekad se koristi za komunikaciju s djedom i bakom, koji predstavljaju zadnju generaciju vezanu za prošlost i kojima je jezik način čuvanja građanskog i kulturnog identiteta. Kuhinja starih Lošinjana nesvjesno prenosi strukturu jezika i običaja od davnih dana.

Ključne riječi: lošinjski dijalekt, kuhinja, Mali Lošinj 